Article

\title{
An Adoptive Threshold-Based Multi-Level Deep Convolutional Neural Network for Glaucoma Eye Disease Detection and Classification
}

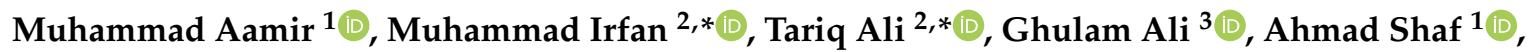 \\ Alqahtani Saeed S ${ }^{4}{ }^{\mathbb{D}}$, Ali Al-Beshri ${ }^{5}$, Tariq Alasbali ${ }^{6}$ and Mater H. Mahnashi ${ }^{7}$ \\ 1 Department of Computer Science, COMSATS University Islamabad Sahiwal Campus, Sahiwal 57000, \\ Pakistan; muhammadaamir@cuisahiwal.edu.pk (M.A.); ahmadshaf@cuisahiwal.edu.pk (A.S.) \\ 2 College of Engineering, Electrical Engineering Department, Najran University, Najran 61441, Saudi Arabia \\ 3 Department of Computer Science, University of Okara, Okara 56300, Pakistan; ghulamali@uo.edu.pk \\ 4 Department of Surgery, Faculty of Medicine, Najran University, Najran 61441, Saudi Arabia; \\ alhafezsaeed@gmail.com \\ $5 \quad$ King Khalid Eye Specialist Hospital, Riyadh 12329, Saudi Arabia; as.beshri@gmail.com \\ 6 Department of Ophthalmology, College of Medicine, Al Imam Mohammad Ibn Saud Islamic \\ University (IMSIU), Riyadh 4233-13317, Saudi Arabia; taalasbali@imamu.edu.sa \\ 7 Department of Pharmaceutical Chemistry, College of Pharmacy, Najran University, Najran 61441, \\ Saudi Arabia; matermaha@gmail.com \\ * Correspondence: irfan16.uetian@gmail.com (M.I.); tariqhsp@gmail.com (T.A.)
}

Received: 18 July 2020; Accepted: 12 August 2020; Published: 18 August 2020

\begin{abstract}
Glaucoma, an eye disease, occurs due to Retinal damages and it is an ordinary cause of blindness. Most of the available examining procedures are too long and require manual instructions to use them. In this work, we proposed a multi-level deep convolutional neural network (ML-DCNN) architecture on retinal fundus images to diagnose glaucoma. We collected a retinal fundus images database from the local hospital. The fundus images are pre-processed by an adaptive histogram equalizer to reduce the noise of images. The ML-DCNN architecture is used for features extraction and classification into two phases, one for glaucoma detection known as detection-net and the second one is classification-net used for classification of affected retinal glaucoma images into three different categories: Advanced, Moderate and Early. The proposed model is tested on 1338 retinal glaucoma images and performance is measured in the form of different statistical terms known as sensitivity (SE), specificity (SP), accuracy (ACC), and precision (PRE). On average, SE of $97.04 \%$, SP of $98.99 \%$, ACC of $99.39 \%$, and PRC of $98.2 \%$ are achieved. The obtained outcomes are comparable to the state-of-the-art systems and achieved competitive results to solve the glaucoma eye disease problems for complex glaucoma eye disease cases.
\end{abstract}

Keywords: ML-DCNN; glaucoma deep-learning; computer vision; convolutional neural network; glaucoma eye disease

\section{Introduction}

Eyes are the most used sensory organ of the human body among the five senses. A significant segment of the mind is utilized in visual processing. Glaucoma, usually caused by increased pressure inside the eye, is the primary root of visual loss over the globe and cannot be rehabilitated. Detection of glaucoma in its beginning is difficult but can be cured [1]. Glaucoma analysis is based on the medicinal history of the patient's family, intraocular pressure (IOP), retinal nerve fiber layer thickness, and changes in optic disc (OD) structure, for example, the distance across, volume, and region. According 
to a study, in 2013 overall 64.3 million people in the population aged 40 to 80 years experienced glaucoma. This number can be exceeded to 76 million by 2020 and 111.8 million by 2040 [2].

The retina layer is composed of roughly one million nerve fibers that organize collectively to make the optic nerves. The start of the optic nerves within the retina layer is termed as an optic disk (OD) or optic nerve head $(\mathrm{ONH})$, which is circular in form and noticeably intense within the retinal images. Symptoms of glaucoma only occur when the disease is slightly advanced; glaucoma is called the silent thief of sight. Therefore, the timely diagnosis of this disease is necessary [3]. Eye screening is a too long and tiresome process because of the keen checkup of each individual patient. To improve the eye screening procedure, a computer aided diagnosis system (CADx) can be used to give more productive results to the patients to distinguish between healthy and infected retinal fundus images as it is hard for oculists to label this distinction accurately.

Over the most recent couple of years, deep learning algorithms have reformed the field of computer vision and are now becoming part of our everyday lives [4]. The machine learning algorithms are appropriate for the analysis of glaucoma. Generally, two primary methodologies utilized for glaucoma identification are segmentation-based and features learning-based approaches. The digital fundus images are utilized for the recognition of glaucoma.

In previous works, the researchers provided a solution for automatic detection and classification of glaucoma via segmentation of the cup to extract the features [5]. To segment the optic disk (OD) and optic cup regions in a sturdy way is a troublesome assignment for the computer-aided system. It requires lots of image processing methods and domain expert knowledge to pick the most biased features. Diagnosing methods on eye fundus images are based on the segmentation of blood vessels and the optic disc regions. Any destruction to the retinal nerve fiber layer (RNFL) is distinguished by utilizing the texture features of retinal fundus images [6].

The objective of this work is to give an automated framework for glaucoma detection through retinal image analysis, which contains phases: a collection of a retinal image database, preprocessing to minimize the amount of noise existing in images, features training, and finally the classification of images as glaucomatous or not. The convolutional neural network (CNN) architecture will be responsible for features learning. The terms accuracy, specificity, sensitivity, and receiver operating characteristic/area under curve (ROC/AUC) have been commonly used as a benchmark for evaluating a diagnosis system. Evaluation of our proposed framework will be performed by using a database containing retinal fundus images of patients from the local hospital.

\section{Materials}

Machine-learning algorithms are appropriate for various complicated image classification problems such as glaucoma disease classification from retinal images [7-9]. Glaucoma is a chronic eye disease caused by eye retinal changes [10] which leads to gradual vision loss and, finally, complete blindness occurs if not diagnosed timely [11,12]. Glaucoma-Deep, a feature-based learning framework was proposed which contains four phases: identification, extraction, optimization, and classification. They utilized a convolutional neural network $(\mathrm{CNN})$ to separate features and supervised a deep-belief network (DBN) to enhance the extracted features and a linear SoftMax classifier to classify amongst ordinary and glaucoma eyes. In [13] tested the C5.0, k-nearest neighbor $(\mathrm{kNN})$, support vector machine (SVM), and random forest (RF) learning algorithms for glaucoma identification in the light of retinal nerve fiber layer (RNFL) density and visual field. Among all, the RF model provided high-quality performance with an accuracy of $0.98 \%$ : a computerized system for glaucoma identification.

In [14] tested 702 retinal images. Initially, RGB images transform to grayscale images by the luminance technique. They utilized a local configuration pattern (LCP) for features extraction, sequential floating forward search (SFFS) for features selection, $t$-test for ranking, and k-nearest neighbor $(\mathrm{kNN})$ classifier for classification. In [15], two datasets, public and private, were used. A 2D Empirical Wavelet Transform (EWT) was utilized to breakdown fundus images and acquire features from 
decomposed EWT for glaucoma identification. Those features were graded via a $t$-test by using a classifier Least-Squares SVM.

In [16], authors covered the segmentation and localization of the optic disc head diagnosis using 3-D datasets, pixel-level glaucomatous changes, and the artificial neural network (ANN) for recognizing the continuation of glaucoma disorder. In [17], a multilayer convolutional neural network deployed and split into four convo layers adjacent to two fully connected layers for glaucoma detection. In [18], RNFL and optic nerve data utilized to test the performance of machine-learning classifiers and RF. The system obtains a 0.877 value of region under the receiver operating characteristic (ROC) via RF. Glaucoma was identification using SVM rather than using a deep-learning algorithm, and separated color, texture features from fundus images [19].

The system was tested on 100 people and got a specificity of $87 \%$ and sensitivity of $100 \%$. Ref. [20] applied feature-based learning on fundus images for glaucoma and choose CNN for feature-learning with an activation function. They utilized normal and glaucomatous eye patterns to evolve for the schooling of Convolutional neural architecture. ORIGA and SCES datasets were used with the area under curve (AUC) values of 0.838 and 0.898 , distinctively, to introduce a model to section optic-disc features extraction in a distinct color style and categorize them by a multilayer perceptron (MLP) framework. Automatic recognition of the eye disorder with image processing techniques was performed through machine-learning classifiers [21].

In [22], a dataset of 1542 fundus images was used, including 786 healthy and 756 glaucoma patients. All these pictures were settled into a $1 \times(240 \times 240 \times 3)$ one-dimensional array to perform logistic regression. The Google Net Inception v3 model with a modified classification layer was used to fulfill their classification needs. They have used an Adam optimizer as an optimization function for backpropagation, and cross-entropy as a loss function. Performance of the developed model and Google Net model depended on the ROC curve by computing specificity and sensitivity of the models. The final version accomplished accuracy and AUC on the test data, training data, and validation data were $87.9 \%$ and $0.94 \%, 92.2 \%$ and $0.98 \%$, and $88.6 \%$ and $0.95 \%$, respectively.

In [23], the presented design resembles the original U-Net; it comprises a contracting (left aspect) path and an expansive (right aspect) path. The contracting way basically rehashes the commonplace engineering of the convolutional part of the classification organizer. On the expansive way, data are converged from layers of the contracting way of suitable goals and layers of broadway of lower goals. Results are reported for freely accessible datasets Drions-db, Rim-One, and Drishti. Analyses results and a visual correlation demonstrate that programmed optic disc division should be possible at the quality aggressive with humans.

In [24] carries out experiments with convolutional neuronal networks to achieve the automatic detection of this disease. For this purpose, they have collected 25 fundus images of normal eye and 19 fundus images of glaucoma patients and then re-trained the Inception v3 convolutional neuronal network. The trained model provides on average a $99 \%$ accuracy for classifying glaucoma and no glaucoma. They still believe that a better predictive model could be generated by retraining the algorithm using more fundus images.

\section{Methodology}

The methodology section defines the overall methodology of the proposed Multi-Level Deep Convolutional Neural Network (ML-DCNN) for glaucoma eye disease detection and classification. The current machine learning and artificial intelligence methods for Glaucoma eye detection have the least number of filters and large time complications. To tackle this issue, Multi-Level Deep Neural Network-based image classification has been proposed. In our work, we chose a features-based learning approach to the multilayer deep Convolutional Neural Network (CNN). We deal with datasets of retinal fundus images. In the proposed research, a Multi-Level Deep Convolutional Neural Network (ML-DCNN) is proposed and implemented for glaucoma eye disease detection and classification. It consists of different CNN layers. It is implemented in two phases: the first one is Detection-Net 
$\mathrm{CNN}(\mathrm{DN}-\mathrm{CNN})$ and the second one is Classification-Net (CN-DCNN) with different layers as their detail explained in Algorithm 1.

\subsection{Multi-Level Deep CNN (ML-DCNN) Architecture}

A Multi-Level Deep Convolutional Neural Network is introduced. It is related to an ordinary neural network having a combination of different neuron layers, learning rates, and other parameters. In this research for the selection of networks, we used four different CNN approaches as shown in Figure 1. The working process of the proposed Multi-Level Deep Convolutional Neural Network is represented in Figure 2. All the CNNs were implemented in two parts as explained below.
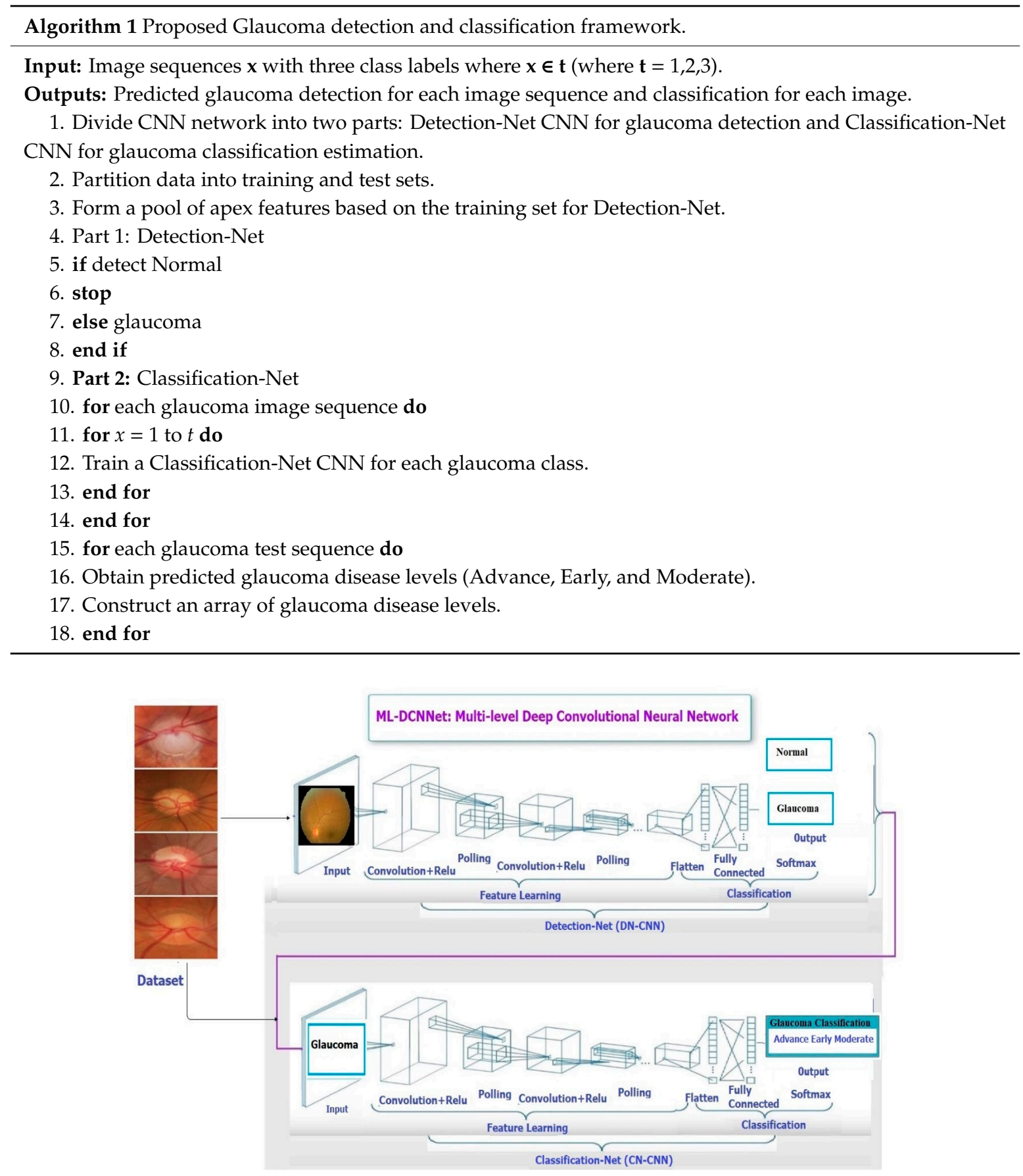

Figure 1. The Architecture of Multi-Level deep Convolutional Neural Network (ML-DCNN). 


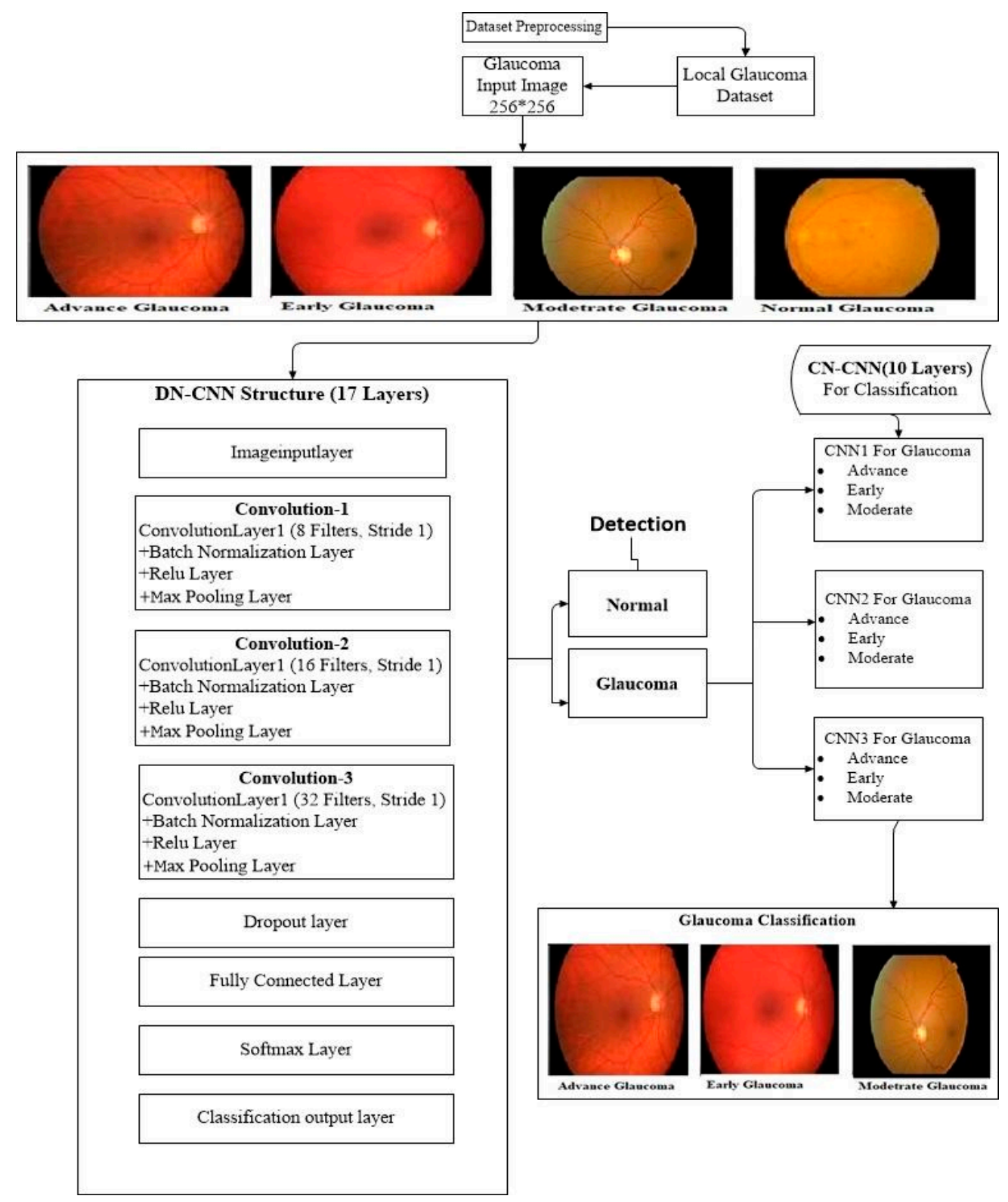

Figure 2. The Representation of Working Process of the (ML-DCNN).

\subsection{Detection-Net CNN (DN-CNN)}

In this portion, the first $\mathrm{CNN}$ is used to detect glaucoma disease. It is known as Detection-Net (DN-CNN) as shown in Figure 3. In this Net, we used a CNN having 17 layers: an image input layer, 3 convolutional layers, 3 batch normalization Layer, 3 ReLu layers, 3 max-pooling layers, a dropout layer, and fully connected SoftMax and classification layers as shown in Table 1. In this table the input image has initially a $256 \times 256$ size with a filter size $3 \times 3$, the number of filters $(\mathrm{k}) 8$, and a stride 1 for the first convolutional layer. In the second convolutional layer, the size decreases to $128 \times 128$; filter size, and stride remains the same with 16 number of filters. In the third convolutional layer, the size is $64 \times 64$ with 32 filters and filter size and stride remain the same. This network, first of all, takes an image as an input in the image input layer and passes through the different CNN layers. The image passes through all the CNN layers to get the features matrix of the image, and at the end of the network classification layer produces the corresponding classification label. This classification is further used as an input in the next classification network to estimate the glaucoma disease level (Advance, Moderate, Early). 


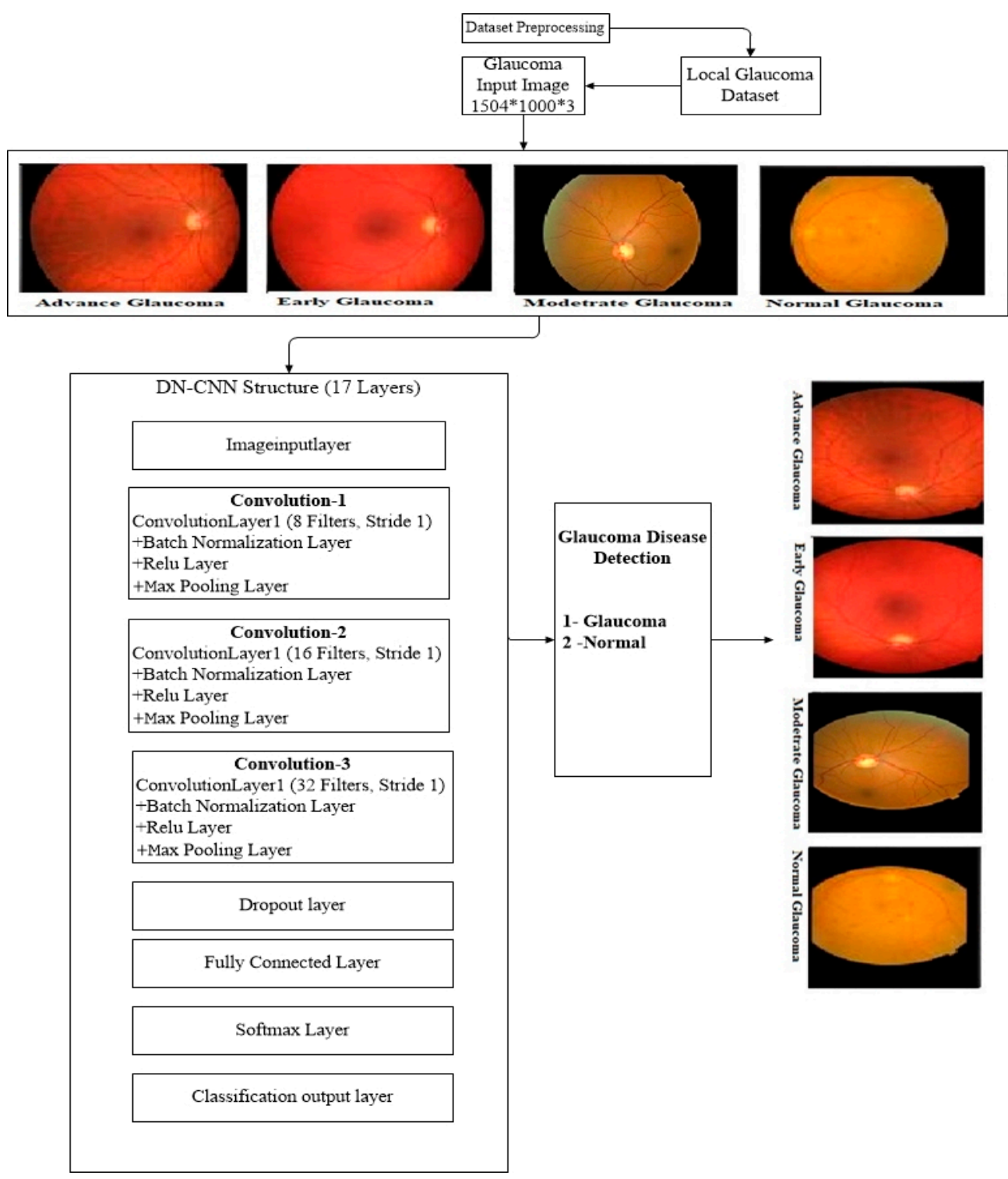

Figure 3. Layer-Wise Representation of the Architecture of Detection-Net-CNN.

Table 1. Description of Layers of Detection-Net Convolutional Neural Network (DN-CNN).

\begin{tabular}{ccc}
\hline \multicolumn{3}{c}{ CNN Layers Description with Learning Rate $=\mathbf{0 . 0 0 1}$ and Epochs $\mathbf{6 0}$} \\
\hline Sr. No & Layers Description & Parameters \\
\hline 1 & Image Input Layer & $256 \times 256$ \\
2 & convolution2dLayer & $256 \times 256,3 \times 3, \mathrm{~K}=8$, stride $=1$ \\
3 & Batch normalization Layer & $3 \times 3$, stride $=1$ \\
4 & Relu Layer & $3 \times 3$, stride $=1$ \\
5 & maxPooling2dLayer & $3 \times 3$, stride $=1$ \\
6 & convolution2dLayer & $128 \times 128,3 \times 3, \mathrm{k}=16$, stride $=1$ \\
7 & Batch normalization Layer & $3 \times 3$, stride $=1$ \\
8 & Relu Layer & $3 \times 3$, stride $=1$ \\
9 & maxPooling2dLayer & $3 \times 3$, stride $=1$ \\
10 & convolution2dLayer & $64 \times 64,3 \times 3, \mathrm{k}=32$, stride $=1$ \\
11 & Batch normalization Layer & $3 \times 3$, stride $=1$ \\
12 & Relu Layer & $3 \times 3$, stride $=1$ \\
13 & maxPooling2dLayer & $3 \times 3$, stride $=1$ \\
14 & Dropout Layer & Dropout $=0.7$ \\
15 & Fully Connected Layer & 2 Classes \\
16 & Softmax Layer & 2 Classes \\
17 & classification Layer & 2 Classes \\
\hline
\end{tabular}




\subsection{Classification-Net CNN (CN-CNN)}

In this part of ML-DCNN architecture, four CNNs are used for glaucoma disease level classification. It is named as Classification-Net CNN (CN-CNN). This network uses classified images from the DN-CNN network to detect glaucoma affected images. The disease level of glaucoma is divided into four phases: advance, early, moderate, and normal, where early describes the beginning of the glaucoma disease, moderate belongs to medium value, advance defines the peak value, and normal explains the no glaucoma disease value. For each stage in the glaucoma detection in the Classification-Net phase, we implemented one $\mathrm{CNN}$ algorithm, so a total of four $\mathrm{CNN}$ architectures are used in this part. The architecture of $\mathrm{CN}-\mathrm{CNN}$ is shown in Figure 4; we used 10 layers with a learning rate of 0.001 and 30 epoch size as explained in Table 2 . In this table, the input image has initially a $256 \times 256$ size with a filter size $3 \times 3$; the number of filters $(\mathrm{k})$ is 8 , and stride is 1 for the first convolutional layer. In the second convolutional layer, the size decreases to $126 \times 126$; filter size, and stride remains the same with 16 filters. In the third convolutional layer, the size is $61 \times 61$ with 32 filters and filter size and stride remains the same.

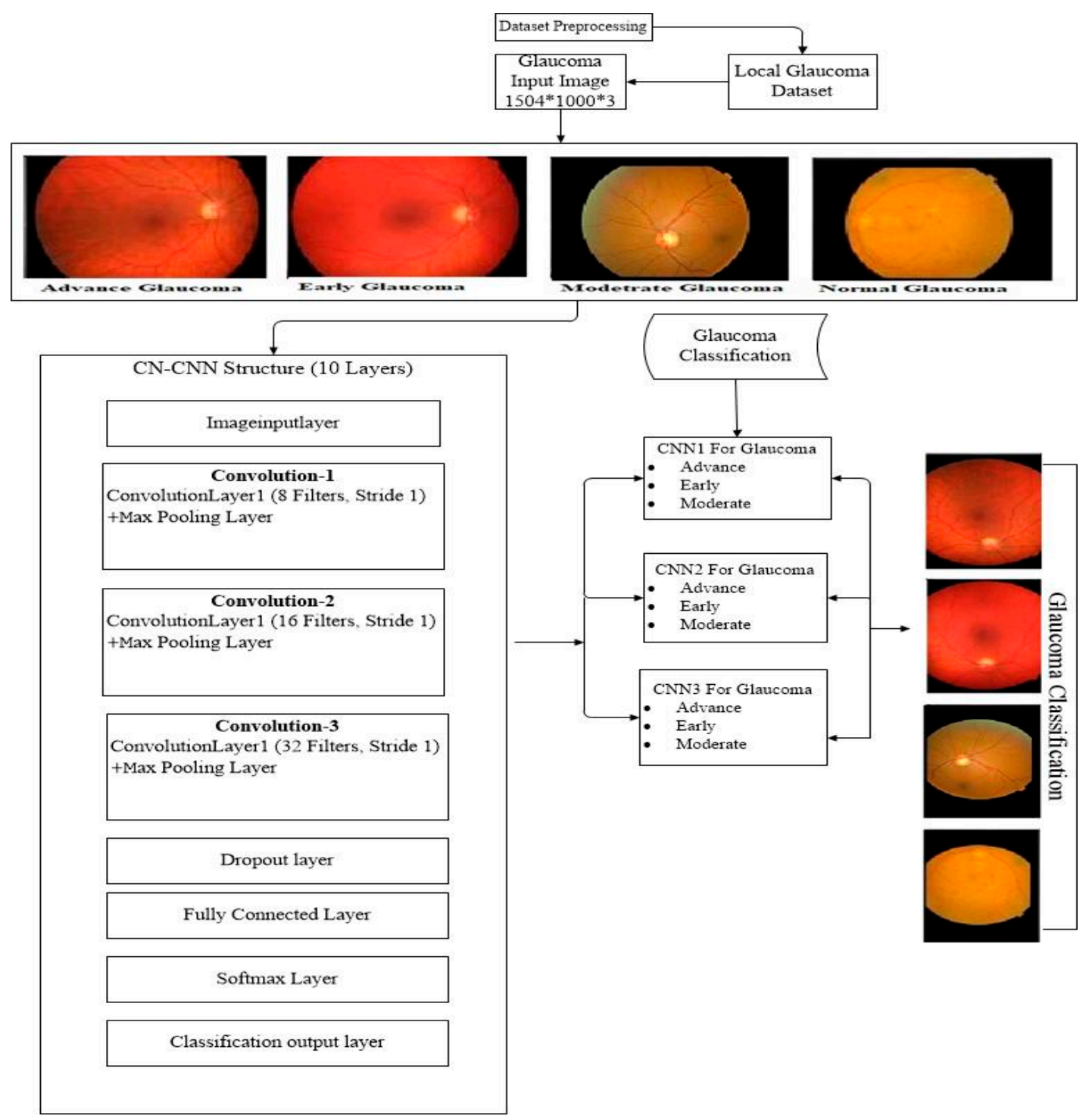

Figure 4. Layer-Wise Representation of the Architecture of Classification-Net-CNN. 
Table 2. Description of Layers of Classification-Net CNN (CN-CNN).

\begin{tabular}{ccc}
\hline & CNN Layers Description with Learning Rate $=\mathbf{0 . 0 0 1}$ and Epochs $=\mathbf{3 0}$ \\
\hline Sr. No & Layers Description & Parameters \\
\hline 1 & Image Input Layer & $256 \times 256$ \\
2 & convolution2dLayer & $256 \times 256,3 \times 3, \mathrm{~K}=8$, stride $=1$ \\
3 & maxPooling2dLayer & $3 \times 3$, stride $=1$ \\
4 & convolution2dLayer & $126 \times 126,3 \times 3, \mathrm{k}=16$, stride $=1$ \\
5 & maxPooling2dLayer & $3 \times 3$, stride $=1$ \\
6 & convolution2dLayer & $61 \times 61,3 \times 3, \mathrm{k}=32$, stride $=1$ \\
7 & maxPooling2dLayer & $3 \times 3$, stride $=1$ \\
8 & Fully Connected Layer & 3 Classes \\
9 & SoftMax Layer & 3 Classes \\
10 & classification Layer & 3 Classes \\
\hline
\end{tabular}

\section{Results and Discussion}

The proposed Multi-Level Deep Convolutional Neural Network (ML-DCNN) was tested on the personal laptop with an Intel Core i7, 2.60 GHz CPU with 8GB RAM in the MATLAB R2018a and different resultant statistical values were calculated.

\section{Dataset Pre-Processing}

The local retinal glaucoma image dataset is pre-processed by an adaptive histogram equalizer to decrease the amount of noise existing in the images. The local retinal glaucoma dataset images are acquired from different private and public resources in different hospitals. The dataset consists of a total of 1338 retinal images. Each image belongs to one of four classes i.e., Normal, Early Glaucoma, Moderate Glaucoma, Advanced Glaucoma. The dataset is a kind of imbalanced dataset because there are about $79 \%$ of images that belong to one class and the remaining $21 \%$ of images belong to the remaining three classes. There are $8.96 \%$ early glaucoma images, $5.98 \%$ moderate glaucoma images, $5.98 \%$ advanced glaucoma images, and $79.08 \%$ normal images (with no glaucoma). The detail of the dataset and its distribution into training, validation, and testing subsets has been shown in Table 3 . To do training and testing on the given dataset, the dataset of 803 images is divided into four categories as advance, early, moderate, and normal with 50,75, 50, and 628 images, respectively, as shown in the third column of Table 4 . The dataset is divided into test, training, and validation datasets. The ratio of testing, validation, and training dataset is $23 \%, 17 \%$, and $60 \%$, respectively, as shown in Table 4 . Three expert clinical assistants were requested to make a difference between four stages of glaucoma eye disease as shown in Figure 5. The majority of voting was used to assign labels to the images where experts disagreed.

Table 3. Distribution of images in the different Groups (Classes) and Subsets.

\begin{tabular}{lcccc}
\hline Group & Total & Train & Validation & Test \\
\hline Advanced Glaucoma & 80 & 50 & 10 & 20 \\
Early Glaucoma & 120 & 75 & 15 & 30 \\
Moderate Glaucoma & 80 & 50 & 10 & 20 \\
Normal & 1058 & 628 & 200 & 230 \\
Total & $\mathbf{1 3 3 8}$ & $\mathbf{8 0 3}$ & $\mathbf{2 3 5}$ & $\mathbf{3 0 0}$ \\
\hline
\end{tabular}


Table 4. Computed Values (\%) of Statistical Measures on Testing Dataset.

\begin{tabular}{clcccc}
\hline \multicolumn{5}{c}{ Detection of Glaucoma Eye Disease } \\
\hline No & Category & Sensitivity (\%) & Specificity (\%) & Accuracy (\%) & Precision (\%) \\
\hline 1 & Advanced Glaucoma & 100.0 & 99.28 & 99.32 & 90.0 \\
2 & Early Glaucoma & 93.54 & 99.62 & 98.9 & 96.6 \\
3 & Moderate Glaucoma & 100.0 & 100.0 & 100.0 & 100.0 \\
4 & Normal & 99.13 & 98.52 & 98.9 & 99.05 \\
& Average & $\mathbf{9 8 . 1 6}$ & $\mathbf{9 9 . 3 5}$ & $\mathbf{9 9 . 2 8}$ & $\mathbf{9 6 . 4 1}$ \\
\hline
\end{tabular}

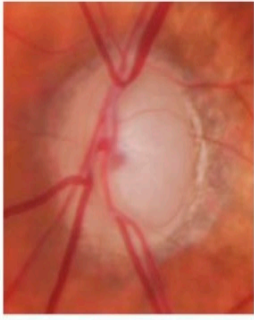

Advance Glaucoma

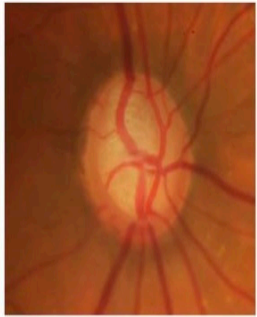

Moderate Glancoma

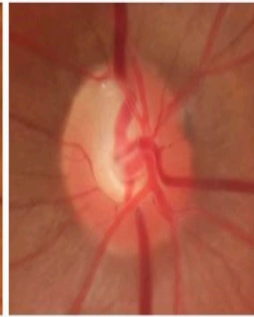

Early Glaucoma

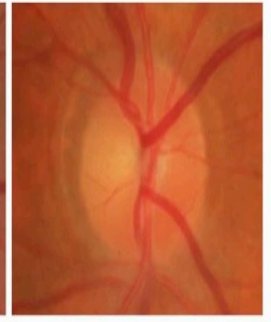

Normal

Figure 5. Glaucoma Stages.

The results of the proposed ML-DCNN are calculated with the help of the following statistical equations.

$$
\begin{aligned}
& \text { Sensitivity }(\mathrm{SE})=\mathrm{TP} /(\mathrm{TP}+\mathrm{FN}) \\
& \text { Specificity }(\mathrm{SP})=\mathrm{TN} /(\mathrm{TP}+\mathrm{FP})
\end{aligned}
$$

$$
\text { Accuracy }(\mathrm{ACC})=(\mathrm{TP}+\mathrm{TN}) /(\mathrm{TP}+\mathrm{FN}+\mathrm{TN}+\mathrm{FP})
$$

Precision $(\mathrm{PRC})=\mathrm{TP} /(\mathrm{TP}+\mathrm{FP})$

Here, TP denotes true positive that is correctly identified between glaucoma images, and TN explains the true negative that identified wrongly classified images. Whereas, false positive (FP) and false-negative (FN) denotes the correctly and wrongly identified classes, as shown in Figure 6.

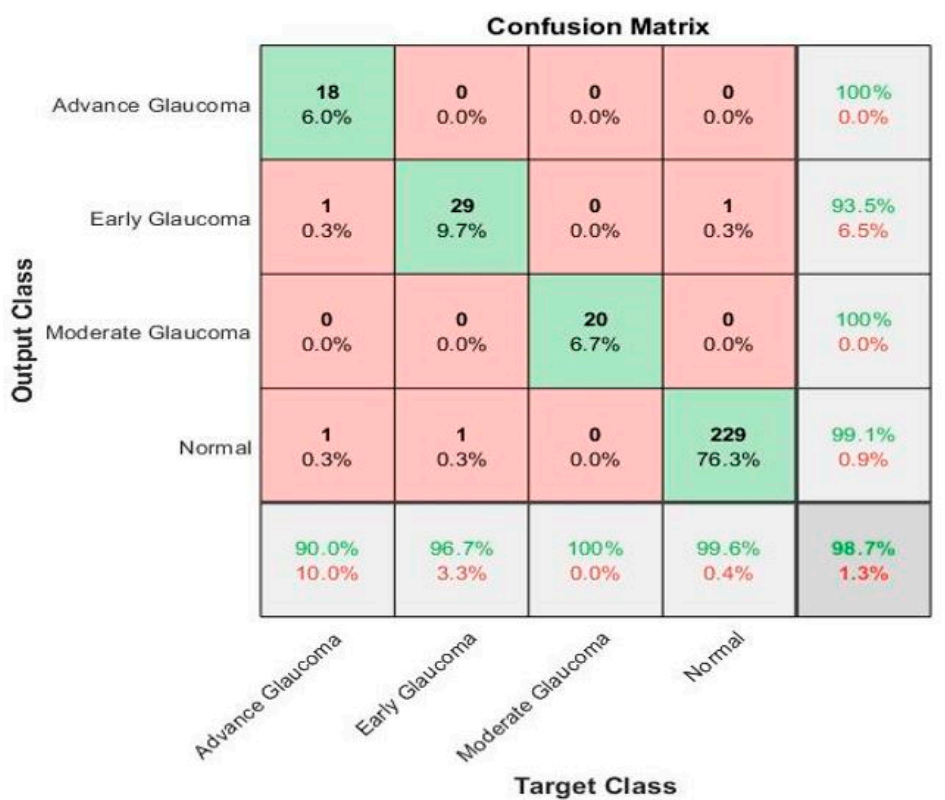

Figure 6. Confusion matrix of the 4-class glaucoma classification using the proposed ML-DCNN model on the Testing Dataset. 
The performance of these parametric equations is calculated on 300 test retinal glaucoma disease images based on a ground truth and proposed ML-DCNN model, as shown in Table 5 and Figure 6.

Table 5. Computed Values (\%) of Statistical Measures on the Local Retinal Glaucoma Image Dataset.

\begin{tabular}{clcccc}
\hline \multicolumn{5}{c}{ Detection of Glaucoma Eye Disease } \\
\hline No & Category & Sensitivity (\%) & Specificity (\%) & Accuracy (\%) & Precision (\%) \\
\hline 1 & Advanced Glaucoma & 96.25 & 99.75 & 99.54 & 96.25 \\
2 & Early Glaucoma & 92.21 & 99.75 & 99.02 & 97.5 \\
3 & Moderate Glaucoma & 100.0 & 100.0 & 100.0 & 100.0 \\
4 & Normal & 99.71 & 96.47 & 99.02 & 99.05 \\
& Average & $\mathbf{9 7 . 0 4}$ & $\mathbf{9 8 . 9 9}$ & $\mathbf{9 9 . 3 9}$ & $\mathbf{9 8 . 2}$ \\
\hline
\end{tabular}

The confusion matrix in Figure 6 shows the results of the recognition rate of different classes on the testing dataset. The rows show the predicted values of the classes and the columns explained the true class values. The diagonal cells show the total number of observations that are correctly classified. The off-diagonal cells explain the incorrect classification of observations. Each cell includes the total number of observations and their percentage. The predicted values' percentage that is correctly and incorrectly classified for each class is presented in the far-right column of the confusion matrix. These values are also called the precision (positive predictive value) and false discovery rate, correspondingly. The correct and incorrect classification percentages of all the classes are explained in the row at the bottom end. These values are frequently called the recall (or true positive rate) and false negative rate, correspondingly. The overall accuracy is shown in the bottom-right cell of the confusion matrix.

Figure 7 shows the validation and training accuracy which is $98.7 \%$, and Figure 8 shows the validation and training loss. Table 4 shows a comparison of the performance of the proposed ML-DCNN algorithm. It shows the performance in the form of an average SE 98.16\%, SP 99.35\%, ACC 99.28\%, and PRC 96.41\%. The proposed ML-DCNN glaucoma model obtained different statistical values for advanced glaucoma, early glaucoma, moderate glaucoma, and normal glaucoma categories as shown in Table 4. The obtained outcomes are comparable to the state-of-the-art systems and achieved competitive results to solve the glaucoma eye disease problems for complex glaucoma eye disease cases.

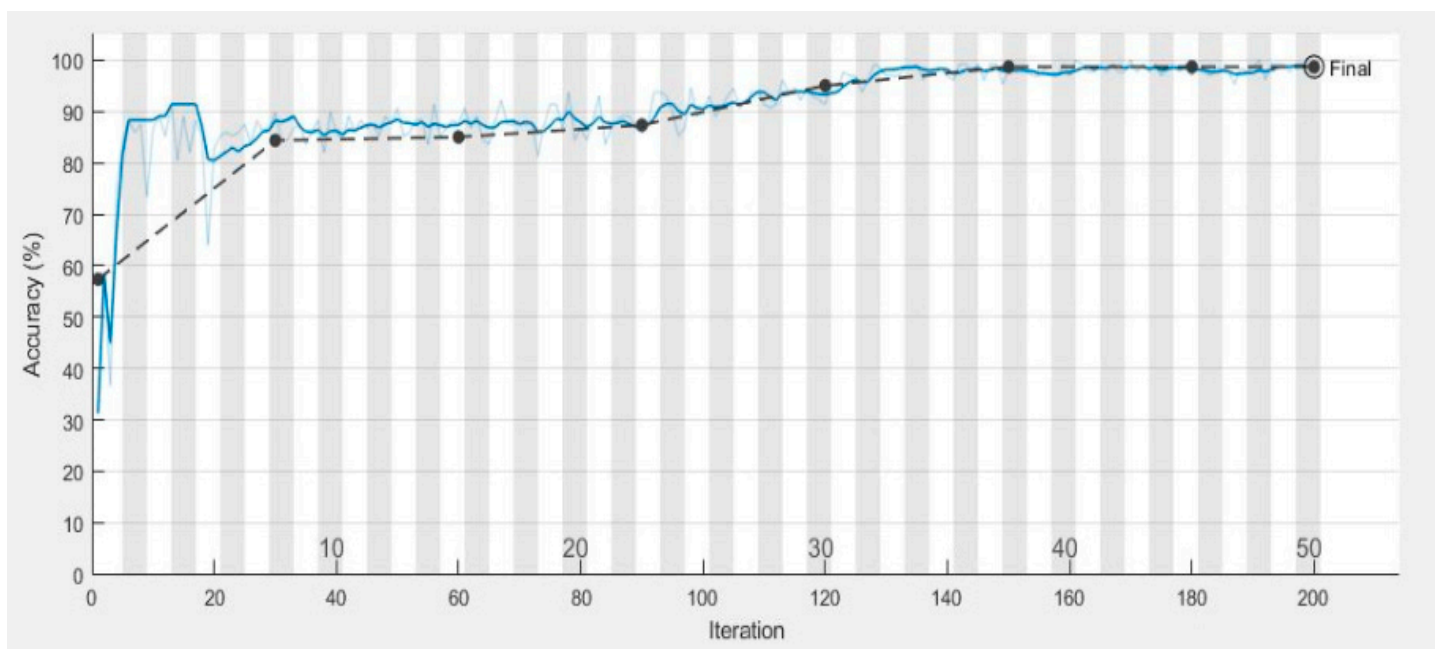

Figure 7. Graphical Representation of Validation and Training Accuracy over the various number of iterations. 


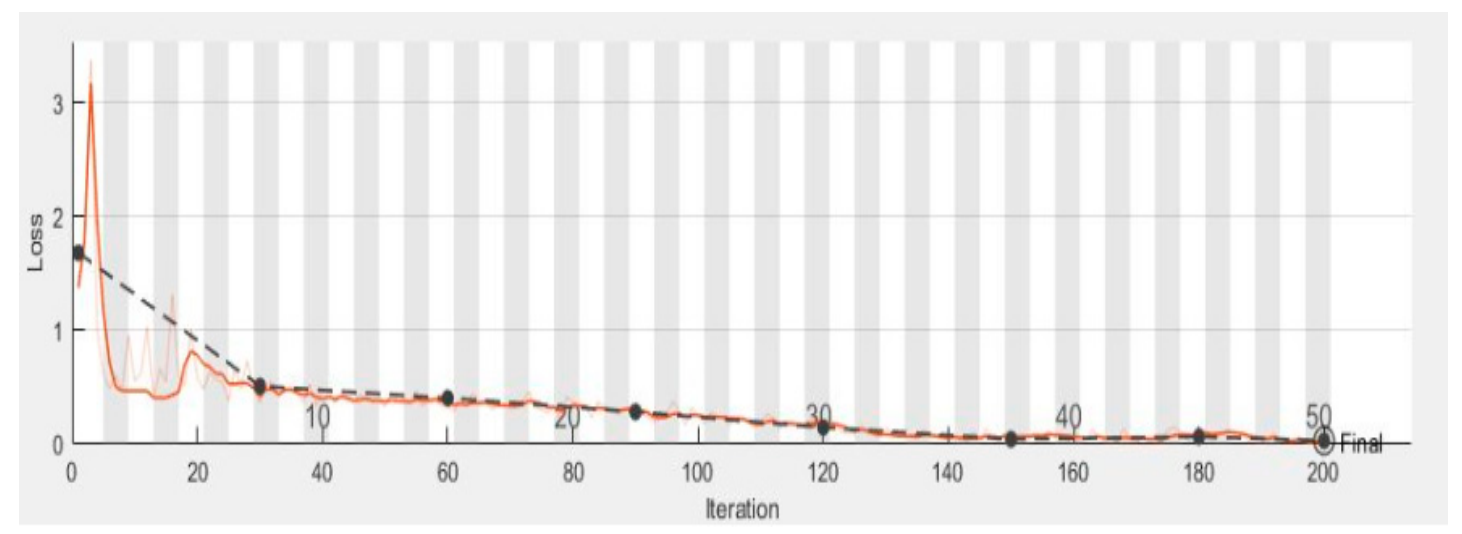

Figure 8. Graphical Representation of Validation and Training Loss Function over the various number of iterations.

Next, we evaluated our proposed framework on the complete dataset of glaucoma images. The confusion matrix in Figure 9 shows the results of the recognition rate of different classes on the local retinal glaucoma image dataset. The rows show the predicted values of the classes and the columns explained the true class values. The diagonal cells show the total number of observations that are correctly classified. The off-diagonal cells explain the incorrect classification of observations. Each cell includes the total number of observations and their percentage. The predicted values' percentages that are correctly and incorrectly classified for each class are presented in the far-right column of the confusion matrix.

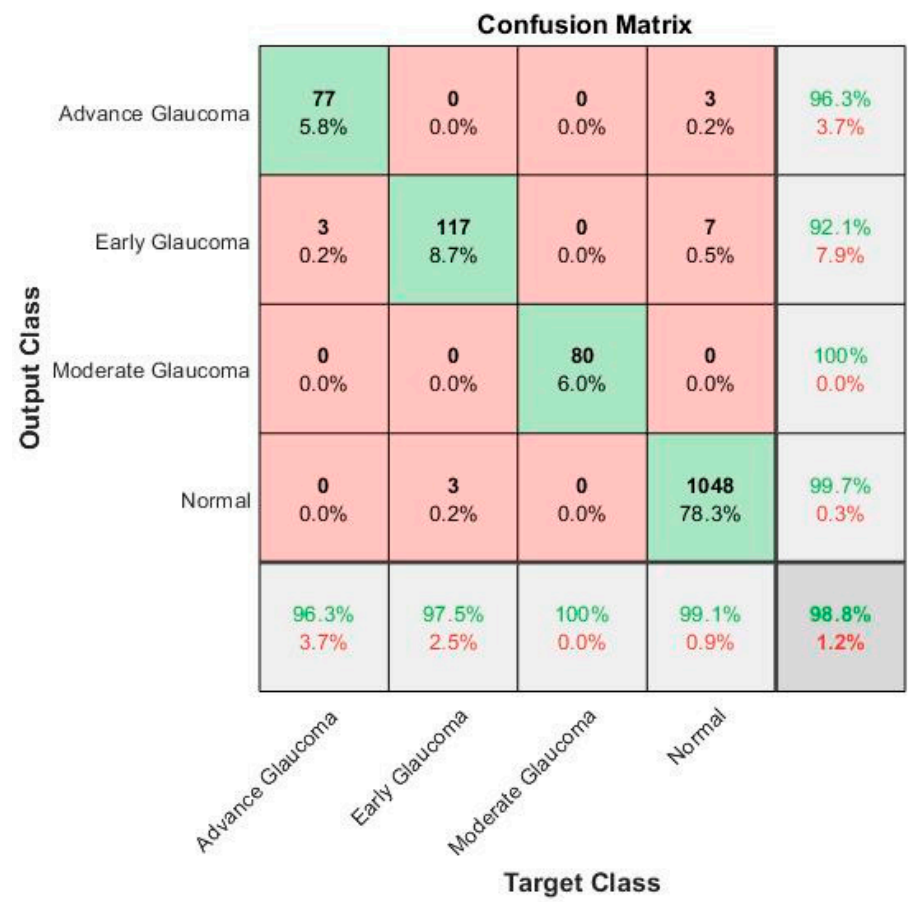

Figure 9. Confusion matrix of the 4-class glaucoma classification using the proposed ML-DCNN Model on the Local Retinal Glaucoma Image Dataset. 
These values are also called the precision (positive predictive value) and false discovery rate, correspondingly. The correct and incorrect classification percentages of all the classes are explained in the row at the bottom end. These values are frequently called the recall (or true positive rate) and the false negative rate, correspondingly. The overall accuracy is shown in the bottom-right cell of the confusion matrix.

Table 5 shows the performance evaluation of the proposed ML-DCNN model on the local retinal glaucoma image dataset. It shows the performance in the form of an average SE 97.04\%, SP 98.99\%, ACC $99.39 \%$, and PRC $98.2 \%$ is observed on a complete dataset. The obtained outcomes are comparable to the state-of-the-art systems and achieved competitive results to solve the glaucoma eye disease problems for complex glaucoma eye disease cases.

In Figure 10 the complete training process is represented graphically. The smooth line shows the training and the dotted line shows the validation of the dataset. In the end, we compared the results of our proposed model with the stat of the art techniques for glaucoma eye disease problem.

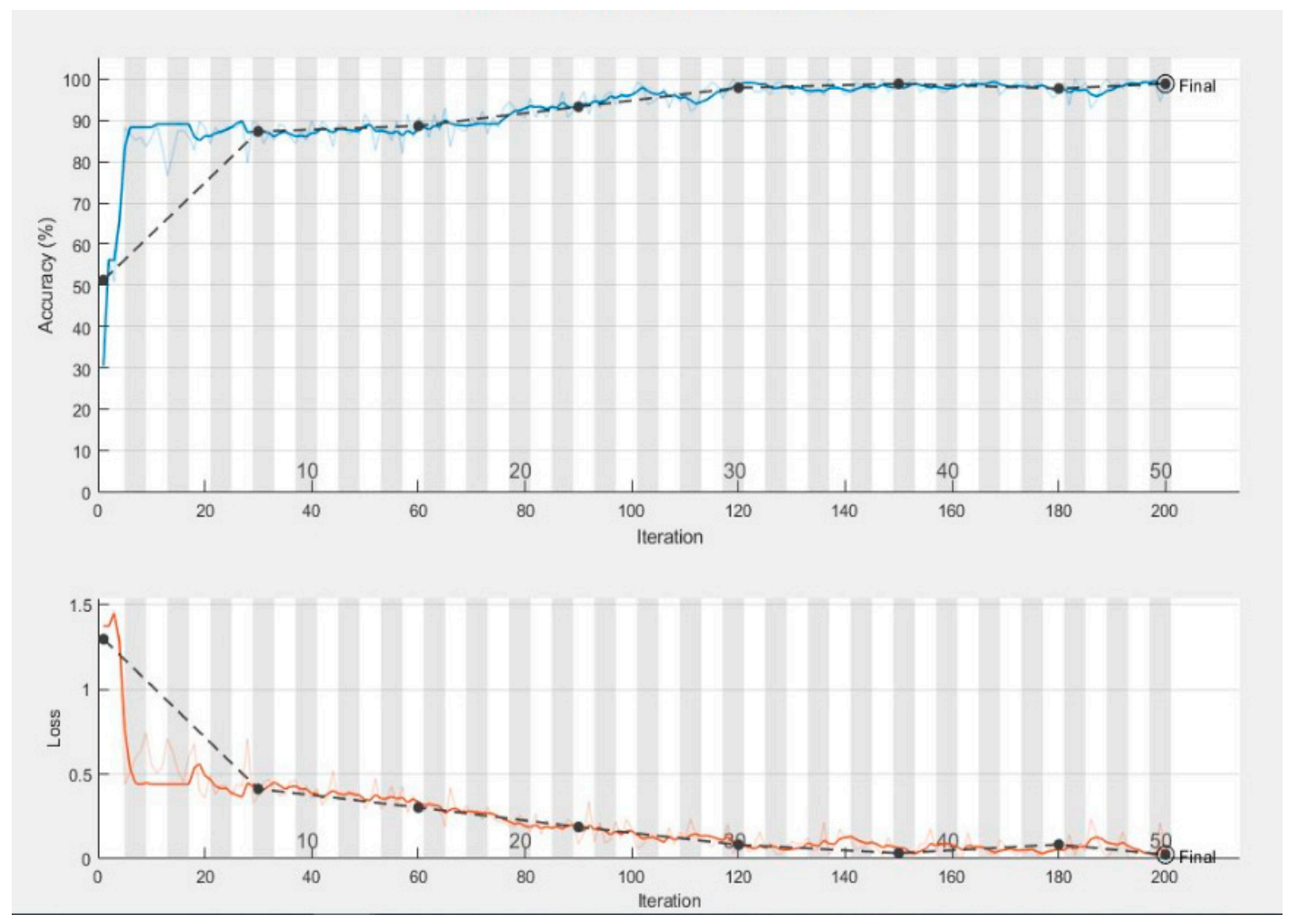

Figure 10. Graphical Representation of complete training process.

Table 6 shows the results of the state-of-the-art systems for the classification of advanced glaucoma, early glaucoma, moderate glaucoma, and normal glaucoma categories of glaucoma eye disease in recent years. As shown in Table 6, the proposed ML-DCNN algorithm gets better statistical values when compared to the other methods for the recognition of eye disease. The reason is that the ML-DCNN glaucoma model works in two parts one for glaucoma detection and the other one is glaucoma classification. 
Table 6. Comparison of Accuracy value (\%) of the proposed technique with the stat of the art techniques.

\begin{tabular}{cccc}
\hline \multirow{2}{*}{ Cited } & \multicolumn{2}{c}{ Comparison of Detection of Glaucoma Eye Disease } & Year \\
\cline { 2 - 4 } & Methodologies & Accuracy (\%) & Year \\
\hline$[17]$ & CNN & 83.00 & 2015 \\
{$[25]$} & Feed-Forward neural network & 92.00 & 2016 \\
{$[19]$} & Support Vector Machine & 87.00 & 2016 \\
{$[21]$} & CNN & 90.00 & 2016 \\
{$[1]$} & Glaucoma-Deep (CNN, DBN d, Softmax) & 99.0 & 2017 \\
{$[10]$} & Semi-Supervised transfer learning for CNN) & 92.4 & 2019 \\
{$[11]$} & OCT Probability map using CNN & 95.7 & 2019 \\
{$[12]$} & AG-CNN & 95.3 & 2019 \\
\hline Proposed ML-DCNN (Advanced, Early, Moderate and Normal Glaucoma) & 99.39 & 2020 \\
\hline
\end{tabular}

\section{Conclusions}

In this paper, the advanced machine deep-learning technique is used on retinal fundus images to diagnose glaucoma affected and normal images. To develop a multi-level deep convolutional neural network (ML-DCNN) for glaucoma detection and classification the CNN framework is implemented on 1338 images to extract features through a multilayer from raw pixel images. The ML-DCNN model is applied in two ways: one for detection of glaucoma as detection-net CNN (DN-CNN); and two for classification of glaucoma, known as classification-net (CN-CNN), into four categories. To evaluate the performance of the ML-DCNN model, the Specificity (SP), Sensitivity (SE), Accuracy (ACC) and Precision (PRC) statistical measures are used, and an average SE of $97.04 \%$, SP of $98.99 \%$, ACC of $99.39 \%$, and PRC of $98.2 \%$ are achieved. The proposed ML-DCNN glaucoma model obtained different statistical values for advanced glaucoma, early glaucoma, moderate glaucoma, and normal glaucoma categories. The obtained outcomes are comparable to the state-of-the-art systems and achieved competitive results to solve the glaucoma eye disease problems for complex glaucoma eye disease cases. The proposed ML-DCNN method performs in a significant way, but in the future this model will be used for other eye diseases.

Author Contributions: M.A. and M.I. have proposed the research conceptualization and methodology. The data gathering is performed by A.S. The technical and theoretical framework is prepared by, G.A. and T.A. (Tariq Ali). The technical review and improvement have been performed by A.S.S and M.H.M. The overall technical support, guidance, and project administration is done by A.A.-B. and T.A. (Tariq Alasbali). All authors have read and agreed to the published version of the manuscript.

Funding: The authors acknowledge the funding by the Ministry of Education and the Deanship of Scientific Research, Najran University. Kingdom of Saudi Arabia, under code number NU/ESCI/19/001.

Acknowledgments: Firstly, the authors acknowledge the Ministry of Education and the Deanship of Scientific Research, Najran University. Kingdom of Saudi Arabia, under code number NU/ESCI/19/001.

Conflicts of Interest: The authors declare no conflict of interest.

Ethical Approval: This article does not contain any studies with human participants or animals performed by any of the authors.

Informed Consent: This article does not contain any implementation of clinically patient data.

\section{References}

1. Abbas, Q. Glaucoma-Deep: Detection of Glaucoma Eye Disease on Retinal Fundus Images Using Deep Learning. Int. J. Adv. Comput. Sci. Appl. 2017, 8, 41-45. [CrossRef]

2. Tham, Y.-C.; Li, X.; Wong, T.Y.; Quigley, H.A.; Aung, T.; Cheng, C.Y. Global prevalence of glaucoma and projections of glaucoma burden through 2040: A systematic review and meta-analysis. Ophthalmology 2014, 121, 2081-2090. [CrossRef] [PubMed] 
3. Maninis, K.-K.; Pont-Tuset, J.; Arbeláez, P.; Van Gool, L. Deep retinal image understanding. In Proceedings of the International Conference on Medical Image Computing and Computer-Assisted Intervention, Athens, Greece, 17-21 October 2016; Springer: Berlin, Germany, 2016.

4. Dos Santos Ferreira, M.V.; De Carvalho Filho, A.O.; De Sousa, A.D.; Silva, A.C.; Gattass, M. Convolutional neural network and texture descriptor-based automatic detection and diagnosis of glaucoma. Expert Syst. Appl. 2018, 110, 250-263. [CrossRef]

5. Raghavendra, U.; Fujita, H.; Bhandary, S.V.; Gudigar, A.; Tan, J.H.; Acharya, U.R. Deep convolution neural network for accurate diagnosis of glaucoma using digital fundus images. Inf. Sci. 2018, 441, 41-49. [CrossRef]

6. Devalla, S.K.; Chin, K.S.; Mari, J.M.; Tun, T.A.; Strouthidis, N.G.; Aung, T.; Thiéry, A.H.; Girard, M.J.A. A deep learning approach to digitally stain optical coherence tomography images of the optic nerve head. Investig. Ophthalmol. Vis. Sci. 2018, 59, 63-74. [CrossRef] [PubMed]

7. Zia, M.S.; Jaffar, M.A. Cross-cultural emotion classification based on incremental learning and LBP-features. Appl. Math. Inf. Sci. 2015, 9, 2651.

8. Butt, U.M.; Husnain, B.; Ahmed, U.; Tariq, A.; Tariq, I.; Butt, M.A.; Zia, M.S. Feature Based Algorithmic Analysis on American Sign Language Dataset. Int. J. Adv. Comput. Sci. Appl. 2019, 10, 583-589. [CrossRef]

9. Zia, M.S.; Hussain, M.; Jaffar, M.A. Incremental Learning-Based Facial Expression Classification System Using a Novel Multinomial Classifier. Int. J. Pattern Recognit. Artif. Intell. 2018, 32, 1856004.

10. Al Ghamdi, M.; Li, M.; Abdel-Mottaleb, M.; Shousha, M.A. Semi-supervised Transfer Learning for Convolutional Neural Networks for Glaucoma Detection. In Proceedings of the ICASSP 2019-2019 IEEE International Conference on Acoustics, Speech and Signal Processing (ICASSP), Brighton, UK, 12-17 May 2019.

11. Thakoor, K.A.; Li, X.; Tsamis, E.; Sajda, P.; Hood, D.C. Enhancing the Accuracy of Glaucoma Detection from OCT Probability Maps Using Convolutional Neural Networks. In Proceedings of the 2019 41st Annual International Conference of the IEEE Engineering in Medicine and Biology Society (EMBC), Berlin, Germany, 23-27 July 2019.

12. Li, L.; Xu, M.; Wang, X.; Jiang, L.; Liu, H. Attention Based Glaucoma Detection: A Large-scale Database and CNN Model. In Proceedings of the IEEE Conference on Computer Vision and Pattern Recognition, Long Beach, CA, USA, 16-20 June 2019.

13. Kim, S.J.; Cho, K.J.; Oh, S. Development of machine learning models for diagnosis of glaucoma. PLoS ONE 2017, 12, e0177726. [CrossRef] [PubMed]

14. Acharya, U.R.; Bhat, S.; Koh, J.E.; Bhandary, S.V.; Adeli, H. A novel algorithm to detect glaucoma risk using texton and local configuration pattern features extracted from fundus images. Comput. Biol. Med. 2017, 88, 72-83. [CrossRef] [PubMed]

15. Maheshwari, S.; Pachori, R.B.; Acharya, U.R. Automated diagnosis of glaucoma using empirical wavelet transform and correntropy features extracted from fundus images. IEEE J. Biomed. Health Inform. 2017, 21, 803-813. [CrossRef] [PubMed]

16. Mary, M.C.V.S.; Rajsingh, E.B.; Naik, G.R. Retinal fundus image analysis for diagnosis of glaucoma: A comprehensive survey. IEEE Access 2016, 4, 4327-4354. [CrossRef]

17. Chen, X.; Xu, Y.; Wong, D.W.K.; Wong, T.Y.; Liu, J. Glaucoma detection based on deep convolutional neural network. In Proceedings of the Engineering in Medicine and Biology Society (EMBC), 2015 37th Annual International Conference of the IEEE, Milan, Italy, 25-29 August 2015.

18. Barella, K.A.; Costa, V.P.; Gonçalves Vidotti, V.; Silva, F.R.; Dias, M.; Gomi, E.S. Glaucoma diagnostic accuracy of machine learning classifiers using retinal nerve fiber layer and optic nerve data from SD-OCT. J. Ophthalmol. 2013, 2013, 7. [CrossRef] [PubMed]

19. Salam, A.A.; Khalil, T.; Akram, M.U.; Jameel, A.; Basit, I. Automated detection of glaucoma using structural and non structural features. Springerplus 2016, 5, 1519. [CrossRef] [PubMed]

20. Chen, X.; Xu, Y.; Yan, S.; Wong, D.W. K.; Wong, T.Y.; Liu, J. Automatic feature learning for glaucoma detection based on deep learning. In Proceedings of the International Conference on Medical Image Computing and Computer-Assisted Intervention, Munich, Germany, 5-9 October 2015; Springer: Cham, Switzerland, 2015.

21. Claro, M.; Santos, L.; Silva, W.; Araújo, F.; Moura, N.; Macedo, A. Automatic glaucoma detection based on optic disc segmentation and texture feature extraction. CLEI Electron. J. 2016, 19, 5. [CrossRef]

22. Ahn, J.M.; Kim, S.; Ahn, K.S.; Cho, S.H.; Lee, K.B.; Kim, U.S. A deep learning model for the detection of both advanced and early glaucoma using fundus photography. PLoS ONE 2018, 13, e0207982. [CrossRef] [PubMed] 
23. Sevastopolsky, A. Optic disc and cup segmentation methods for glaucoma detection with modification of U-Net convolutional neural network. Pattern Recognit. Image Anal. 2017, 27, 618-624. [CrossRef]

24. Liu, S.; Graham, S.L.; Schulz, A.; Kalloniatis, M.; Zangerl, B.; Cai, W.; Gao, Y.; Chua, B.; Arvind, H.; Grigg, J.; et al. A deep learning-based algorithm identifies glaucomatous discs using monoscopic fundus photographs. Ophthalmol. Glaucoma 2018, 1, 15-22. [CrossRef] [PubMed]

25. Asaoka, R.; Murata, H.; Iwase, A.; Araie, M. Detecting preperimetric glaucoma with standard automated perimetry using a deep learning classifier. Ophthalmology 2016, 123, 1974-1980. [CrossRef] [PubMed]

(C) 2020 by the authors. Licensee MDPI, Basel, Switzerland. This article is an open access article distributed under the terms and conditions of the Creative Commons Attribution (CC BY) license (http://creativecommons.org/licenses/by/4.0/). 